\title{
Políticas e programas para o enfrentamento da violência doméstica contra as crianças: Algo novo na pandemia da COVID-19?
}

\author{
Letícia Harumi Shimabukuro', Karen Namie Sakata-So', Lucimara \\ Fabiana Fornari', Emiko Yoshikawa Egry ${ }^{1}$ \\ ${ }^{1}$ Departamento de Enfermagem em Saúde Coletiva, Universidade de São Paulo, Brasil \\ leticiashm@usp.br; knsakata@usp.br; lucimarafornari@usp.br; emiyegry@usp.br \\ https://orcid.org/0000-0002-3508-9354; https://orcid.org/0000-0001-5584-4161; \\ https://orcid.org/0000-0002-8655-6549; https://orcid.org/0000-0003-0974-0315
}

\begin{abstract}
Resumo: Introdução: A violência doméstica contra crianças é um fenômeno prevalente na sociedade. Na pandemia da COVID-19, dadas as medidas de distanciamento social e fechamento temporário das escolas, houve o agravamento do problema. Objetivos: Identificar as políticas e os programas para o reconhecimento das necessidades de saúde com enfoque na violência doméstica infantil na pandemia da COVID-19 e verificar a potência do estudo qualitativo-documental para a análise do fenômeno. Métodos: Trata-se de um estudo qualitativo do tipo documental. O cenário foi um município da Região Sul do Brasil. A coleta dos dados foi realizada de outubro de 2020 a janeiro de 2021, em sites oficiais referentes ao tema. O tratamento dos dados encontra-se em desenvolvimento e será realizado por meio da técnica de análise de conteúdo temática com o apoio do software webQDA. Resultados: Os resultados preliminares mostraram que, durante a pandemia de COVID-19, houve redução nas denúncias de violência contra crianças e adolescentes, além da dificuldade de acesso às redes de apoio, ocasionando a subnotificação dos registros. Foram selecionados 13 documentos referentes às políticas e programas, alguns mundiais e outros emanados de distintas esferas de governo brasileiro. Todos eles carecem de uma definição de metas a serem alcançadas no enfrentamento da violência doméstica contra as crianças durante a pandemia. Duas categorias empíricas foram destacadas: impacto da pandemia na notificação dos casos e caracterização da violência nas políticas e programas. Conclusões: $O$ estudo documental mostra-se um recurso potente para identificar a natureza dos programas e políticas referentes ao enfrentamento do fenômeno em estudo. Ainda que preliminar, as políticas e programas precisam ser mais assertivas para o enfrentamento da violência contra crianças, expostas diariamente aos agressores, sem intermédio das redes de apoio, diminuindo a probabilidade de acompanhamento e notificação dos casos.
\end{abstract}

Palavras-chave: Maus-Tratos Infantis; Violência Doméstica; Política Pública; Pandemias; Pesquisa Qualitativa.

Policies and Programs to Tackle Domestic Violence Against Children: Something New in the COVID-19 Pandemic?

Abstract: Introduction: Domestic violence against children is a prevalent phenomenon in society. In the COVID-19 pandemic, given the social distance and temporary closure of schools, the problem worsened. Objectives: To identify policies and programs for the recognition of health needs with a focus on domestic violence against children in the COVID19 pandemic and to verify the power of qualitative and documentary studies for the analysis of the phenomenon. Methods: This is a qualitative, documentary study. The scenario was a municipality in the Southern Region of Brazil. Data collection was carried out from October 2020 to January 2021, on official websites related to the topic. Data processing is under development and will be carried out using thematic content analysis technique with the support of the webQDA software. Results: Preliminary results showed that, during the COVID-19 pandemic, there was a reduction in reports of violence against children and adolescents, in addition to the difficulty of accessing support networks, causing underreporting of records. Thirteen documents related to policies and programs were selected, some worldwide and others emanating from different spheres of government - municipal, state and federal. All of them lack a definition of goals to be achieved in tackling domestic violence against children during the pandemic. Two empirical categories were highlighted: impact of the pandemic on the notification of cases and characterization of violence in policies and programs. Conclusions: The documentary study proves to be a powerful resource to identify the nature of programs and policies related to coping with the phenomenon under study. Although preliminary, policies and programs need to be more assertive to face violence against children, exposed daily to aggressors, without the support networks, reducing the likelihood of monitoring and reporting cases.

Keywords: Child Abuse; Domestic Violence; Public Policy; Pandemics; Qualitative Research 


\section{Introdução}

Em dezembro de 2019 o novo coronavírus (SARS-CoV-2) foi identificado pela primeira vez na China. Em fevereiro de 2020, houve o alerta de casos suspeitos da doença em outros oito países, e, no final do mês, foi confirmado o primeiro caso de coronavírus do Brasil. Em março de 2020, a Organização Mundial da Saúde declarou o estado de pandemia devido à rápida disseminação geográfica da doença num curto intervalo de tempo (OMS, 2020).

Na pandemia da COVID-19, a restrição de circulação de pessoas, o fechamento das escolas, a ameaça iminente de diminuição da renda financeira, a incerteza sobre o futuro e o excesso de informações midiáticas, aumentaram o nível de estresse e ansiedade em famílias (Cluver, et al., 2020). Identificou-se um aumento nas ligações para linhas de ajuda sobre abuso infantil, e paradoxalmente, houve redução no número de casos registrados nos serviços de proteção à criança (WHO, 2020).

Verificou-se que $66 \%$ dos países relataram uma interrupção em algum serviço relacionado à violência contra crianças. Em contrapartida, apenas $12 \%$ dos países relataram uma não interrupção. Entre esses serviços, incluem-se linhas de apoio à proteção infantil ou call centers, acesso de crianças e famílias ao bem-estar social, programas de prevenção à violência, serviços de gestão de casos ou revisão de caminhos para prevenir violência contra criança, visitas domiciliárias a crianças e mulheres vítimas de abuso (UNICEF, 2020).

A OMS, em publicação com a End Violence, convocou os governos e a comunidade internacional para proteger as crianças dos riscos de violência por meio de uma resposta coletiva, incluindo saúde mental e apoio psicossocial, proteção social e cuidado para as crianças mais vulneráveis e para crianças em instituições de acolhimento (WHO, 2020). No Brasil, o atendimento às vítimas é um serviço essencial e não poderá ser interrompido enquanto durar o estado de calamidade pública causado pelo COVID-19 (Platt, Guedert, \& Coelho, 2020).

Nesta perspectiva, as perguntas científicas do presente estudo foram: Quais são as políticas e os programas de um município brasileiro para o reconhecimento das necessidades em saúde com enfoque na violência doméstica infantil? Quais são as potencialidades do estudo qualitativo-documental para a análise do objeto de estudo? Diante disso, o estudo objetivou identificar as políticas e os programas de um município brasileiro para o reconhecimento das necessidades em saúde com enfoque na violência doméstica contra a criança na pandemia de COVID-19 e verificar a potência do estudo qualitativo-documental para a análise do fenômeno.

\section{Métodos}

Trata-se de um estudo qualitativo do tipo documental. A pesquisa qualitativa envolve problemas de pesquisa que visam compreender as condições que acentuam as desigualdades sociais entre os indivíduos na sociedade. Além disso, a coleta, análise e apresentação dos resultados buscam enfatizar a postura interpretativa do pesquisador (Creswell, 2014).

Ao considerar a diversidade das pesquisas de abordagem qualitativa, as autoras optaram pelo tipo documental dada a natureza do fenômeno e objeto deste estudo. A pesquisa documental utiliza materiais sem prévio tratamento analítico proveniente de diversas fontes.

Diferencia-se da pesquisa bibliográfica na medida em que utiliza como fontes estudos científicos que já passaram por uma análise (Gil, 2017).

$\mathrm{Na}$ pesquisa documental a escolha do documento a ser analisado não é aleatória e dependerá do fenômeno e da pergunta de pesquisa a ser respondida com base num dado referencial teórico (Kripka, Scheller, \& Bonotto, 2015). 
Portanto, para o presente estudo, os documentos escolhidos foram as políticas e os programas referentes ao enfrentamento da violência doméstica infantil no âmbito de município de Curitiba, do estado do Paraná e do Brasil durante a pandemia da COVID19.

\subsection{Referencial Teórico Metodológico}

Este estudo está fundamentado na Teoria da Intervenção Práxica da Enfermagem em Saúde Coletiva (TIPESC), construída para compreender as contradições da realidade objetiva da Enfermagem em Saúde Coletiva entendida como campo teórico e de práticas. Trata-se de uma teoria de Enfermagem, assentada na visão de mundo materialista histórico-dialética, que procura construir uma intervenção de Enfermagem através de uma metodologia dinâmica, dialetizada e participativa (Egry, 1996).

A violência é uma construção social e histórica, que pode aumentar ou diminuir de acordo com sua reprodução na sociedade nos níveis coletivos e individuais (Minayo, 2006). É um fenômeno complexo e de múltiplas causas. Entre as suas manifestações, há a violência estrutural, social e interpessoal (Ministério dos Direitos Humanos, 2018).

A violência interpessoal intrafamiliar ocorre nas relações hierárquicas e inter geracionais, atingindo mais as crianças em razão da fragilidade física e emocional. Consiste em formas agressivas da família se relacionar e se comunicar, por meio do uso da violência como solução de conflito e como estratégia de educação, um padrão de convivência que pode ser estabelecido desde as gerações anteriores. Este cenário hostil prejudica o desenvolvimento da criança, uma vez que inclui falta de cuidados básicos e negligência (Ministério da Saúde, 2010; Silva, Oliveira, 2020).

A violência doméstica distingue-se da intrafamiliar, pois envolve outros membros do grupo social sem laços de parentesco, e abrange os conceitos de abuso físico, sexual e psicológico, a negligência e o abandono. Assim, ela constitui ação ou omissão pelo adulto responsável pelo cuidado, a qual resulta em dano ao desenvolvimento físico, emocional, intelectual e social da criança (Ministério dos Direitos Humanos, 2018).

Além do comportamento individual ou familiar, há dimensões socioeconômicas, que repercutem em toda a comunidade, consequentemente, influenciando no cotidiano das crianças e suas famílias. Portanto, as redes sociais de apoio são importantes para o trabalho e a interação com a família. Infelizmente, nota-se que há estados negligentes em políticas públicas e ações efetivas, pois um Estado presente e ativo promove a defesa e a proteção à infância, enquanto órgãos responsáveis e a sociedade civil monitoram leis e campanhas de combate à violência infantil. Dessa forma, há uma corresponsabilidade do Estado, da família e da sociedade em assegurar os direitos de crianças e adolescentes, assim como, no caso de violação desses direitos, os três são corresponsáveis pelo enfrentamento e superação do problema (Ministério da Saúde, 2010).

A partir de um ciclo de identificação de problemas sociais, iniciam-se as políticas, as quais são dependentes da capacidade técnico-administrativa da gestão pública, assim como das recomendações sociais e do orçamento público. Planeja-se uma agenda de acordo com a urgência e a prioridade governamental e, em seguida, elaboram-se alternativas possíveis para que haja tomada de decisão pela solução mais viável.

Depois de delineadas e formuladas, as políticas públicas são desenvolvidas em planos, programas, projetos, bases de dados ou sistema de informação e pesquisas, inclusive algumas requerem nova legislação.

Após a política pública ser implementada, são necessárias a avaliação e 0 acompanhamento constante pelos gestores e pela sociedade civil, a fim de avaliar a eficiência, eficácia e efetividade em relação ao problema inicial (CLP Liderança Pública, 2019). 
É inegável a importância das políticas públicas no cenário atual, porém deve-se pensar na pandemia como uma situação heterogênea. Apesar de chegar a todas as pessoas, atinge mais algumas do que outras, pois há fatores determinantes de maior exposição à violência, como a situação de vulnerabilidade familiar, a ausência de políticas de geração de renda, habitação e saneamento básico. Diante desse contexto, a desigualdade aumenta, inclusive em relação à violência doméstica (Ministério dos Direitos Humanos, 2018).

\subsection{Cenário da Pesquisa}

O cenário da pesquisa foi o município de Curitiba, localizado no estado do Paraná, Região Sul do Brasil.

A Rede de Proteção à Criança e ao Adolescente em Situação de Risco para a Violência, em 2015, contabilizou 4.421 crianças e adolescentes de Curitiba, das quais $86,7 \%$ das notificações realizadas no município referiam-se à violência intrafamiliar, com ocorrência no ambiente doméstico, no âmbito das relações familiares, na qual prevalece a negligência $(67,2 \%)$, seguida da violência física $(11,2 \%)$ e da sexual $(6,4 \%)$ (Curitiba, 2016; SMS, 2017).

Em 2019, de acordo com o Sistema de Informação de Agravos de Notificação (Sinan), houve 5.725 notificações de violência interpessoal/autoprovocada contra crianças e adolescentes residentes no município de Curitiba. Ao analisar a última década, desde 2010, observa-se um aumento de 44,7\%, apontando para a melhor capacitação e sensibilização, por serviços e profissionais, em notificar casos suspeitos ou confirmados (Wollmann, Sato, Moreira, Cortiano, \& Rosa, 2020).

\subsection{Coleta de Dados}

Para coleta de dados foi realizada uma busca por políticas e programas referentes ao enfrentamento da violência doméstica infantil no período da pandemia da COVID-19. Para seleção dos documentos, foram consultados 17 sites oficiais no âmbito mundial, nacional, estadual e municipal:

a) Mundial: Fundo das Nações Unidas para a Infância (Unicef), The Alliance for Child Protection in Humanitarian Action e Organização Mundial da Saúde (OMS).

b) Nacional: Unicef Brasil, Ministério da Saúde, Conselho Nacional dos Direitos da Criança e do Adolescente (Conanda), Instituto de Pesquisa Econômica Aplicada (Ipea), Childhood Brasil, Fundação Oswaldo Cruz (Fiocruz) e Fundação Abrinq.

c) Estadual: Ministério Público do Paraná, Cadê Paraná, Secretaria de Saúde do Paraná, Portal de Transparência do Governo do Paraná, Defensoria Pública do Paraná e Fundação Ação Social Paraná.

d) Municipal: Secretaria de Saúde de Curitiba.

Os critérios para inclusão dos documentos foram: políticas ou programas construídos pelo município de Curitiba referentes ao tema do enfrentamento da violência doméstica contra a criança e medidas para a contenção da COVID-19 durante a pandemia relacionadas à violência doméstica; e políticas ou programas de âmbito estadual, nacional ou mundial que estivessem relacionados às políticas e aos programas do município de Curitiba no que se refere ao tema em investigação. Foram excluídas as políticas e programas que não estavam associadas à pandemia da COVID-19.

As buscas dos documentos foram realizadas no período de outubro de 2020 a janeiro de 2021. As políticas e os programas foram acessados no campo de busca de cada um dos sites investigados, por meio dos termos: violência doméstica; violência contra crianças; pandemia; políticas e programas. Todas as informações obtidas foram lidas, considerando os critérios de inclusão para, em seguida, selecionar as que fariam parte do estudo. 
Os dados foram extraídos com auxílio de um instrumento semiestruturado elaborado pelas autoras do estudo a fim de padronizar a coleta das informações dos documentos relativas ao título do documento, ano de publicação, nível de governo, fonte do documento, tipo do documento, entendimento da violência, setor de abrangência, objetivo da política ou programa, público-alvo, estratégias de ação, metas, formas de avaliação e recomendação para a prevenção ou enfrentamento da violência doméstica contra crianças. O instrumento foi construído a partir do objetivo do estudo e no formato de planilha Excel para, posteriormente, facilitar o manejo dos dados a partir do recurso de filtros e classificações.

As políticas e programas selecionados foram salvos no formato Portable Document Format (PDF). Em seguida, houve a criação de uma pasta compartilhada na plataforma Google Drive. Os documentos foram divididos entre as quatro autoras do estudo para leitura e preenchimento do instrumento semiestruturado. No final do preenchimento do instrumento, houve uma reunião online por meio da plataforma Google Meet para discussão das divergências relacionadas à inclusão dos documentos e estabelecimento de consenso.

\subsection{Análise de Dados}

Os dados serão analisados por meio da análise de conteúdo temática proposta por Bardin (2011), que prevê as seguintes etapas: pré-análise, exploração de material, tratamento dos resultados, interpretação e inferência.

Além disso, a análise de conteúdo temática será apoiada pelo software webQDA, que permite tratar os dados provenientes de diversas fontes. Está estruturado em quatro sistemas: fontes, espaço destinado para inserção dos dados; codificação, responsável pela criação das categorias descritivas e empíricas; questionamento, dispõe de ferramentas que auxiliam a aprofundar a análise dos dados; e, gestão, permite a organização do processo de trabalho (Minayo, \& Costa, 2019).

O instrumento semiestruturado preenchido será inserido no webQDA por meio da ferramenta de importação automática do sistema de fontes internas. Os dados descritivos relativos ao ano de publicação, nível de governo, setor de abrangência, público-alvo, fonte e tipo do documento serão codificados automaticamente. Os dados empíricos associados ao entendimento da violência, o objetivo da política ou programa, as estratégias de ação, as metas, as formas de avaliação e recomendação para a prevenção ou enfrentamento da violência doméstica contra crianças serão codificados pela primeira autora e validados pela segunda. Após a emergência das categorias e subcategorias empíricas, os dados serão submetidos ao questionamento por meio das matrizes.

Ressalta-se que os dados empíricos serão analisados a partir das categorias sociais de gênero e geração, sustentadas pela TIPESC e que permitem discutir a essência do fenômeno em estudo para além da aparência descritiva.

As categorias sociais (classe social, gênero, geração e raça) têm predominância variável tanto de acordo com a subjetividade construída socialmente quanto à articulação entre elas. Embora sejam características naturais, compartilham significados econômicos, culturais e políticos, os quais se organizam de forma hierárquica, viabilizando privilégios e desigualdades que compõem a rede complexa de poder e dominação. Logo, as categorias devem ser visualizadas juntas, pois em conjunto explicam as condições de vida e de saúde coletiva (Egry, Fonseca, \& Oliveira, 2013).

\section{Resultados e Discussão}

Em relação às políticas e programas que abordaram a violência doméstica contra as crianças no período da pandemia da COVID-19, foram identificados 13 documentos. A maior parte foi classificada como notas técnicas $(n=8)$, seguida por cartilhas $(n=2)$. Os restantes trata-se de nota de orientação, carta pública e recomendação. 
No que se refere à fonte dos dados, seis documentos correspondiam ao nível estadual, cinco ao nível nacional e dois ao nível internacional. Ressalta-se a ausência de documentos no nível municipal.

Sendo dados que ainda estão em fase de análise, foram selecionadas duas categorias empíricas para serem apresentadas como resultados parciais até o momento desta publicação: Impactos da pandemia na notificação dos casos de violência contra a criança e Caracterização da violência doméstica contra as crianças nos programas e políticas públicas durante a pandemia de COVID-19.

\subsection{Impacto da Pandemia na Notificação dos Casos de Violência contra a Criança}

O distanciamento social traz riscos relacionados ao próprio isolamento dentro do lar, pois há um "paradoxo da pandemia": enquanto governos solicitam que as pessoas fiquem em casa em combate ao COVID-19, para indivíduos vulneráveis e que já vivenciavam situações de violência intrafamiliar, o domicílio pode não ser o local mais seguro e protegido (Bradbury-Jones, Isham, 2020). Isolar é uma tática de controle bastante utilizada pelos agressores, uma vez que, sem a rede social de apoio, conseguem esconder as violações e garantir o silêncio das vítimas (The Alliance for Child Protection in Humanitarian Action, 2020; WHO Global, 2020).

No que se refere ao impacto da violência contra crianças brasileiras na pandemia de COVID-19, o Painel de Dados da Ouvidoria Nacional dos Direitos Humanos revelou que, durante o ano de 2020, ocorreram 95.247 denúncias e 368.333 violações contra crianças e adolescentes, sendo o grupo vulnerável com maior número de denúncias relatadas no país. Deste total, o Paraná é responsável por 3.753 denúncias (3,94\%) e 14.411 violações $(3,91 \%)$ e o município de Curitiba, por 1.026 denúncias (2,91\%) e 4.088 violações $(1,10 \%)$. É válido lembrar que uma denúncia pode conter uma ou mais violações de direitos humanos (Ministério da Família, da Mulher e dos Direitos Humanos, 2020).

Conforme mostra a Figura 1, numa análise do ano de 2020, verifica-se uma queda brusca das denúncias totais, sobretudo de março para abril, quando iniciaram as medidas de distanciamento social no Brasil. Em nível municipal, ao final do ano, nota-se uma quantidade inferior à metade do número de denúncias em relação ao início do ano.
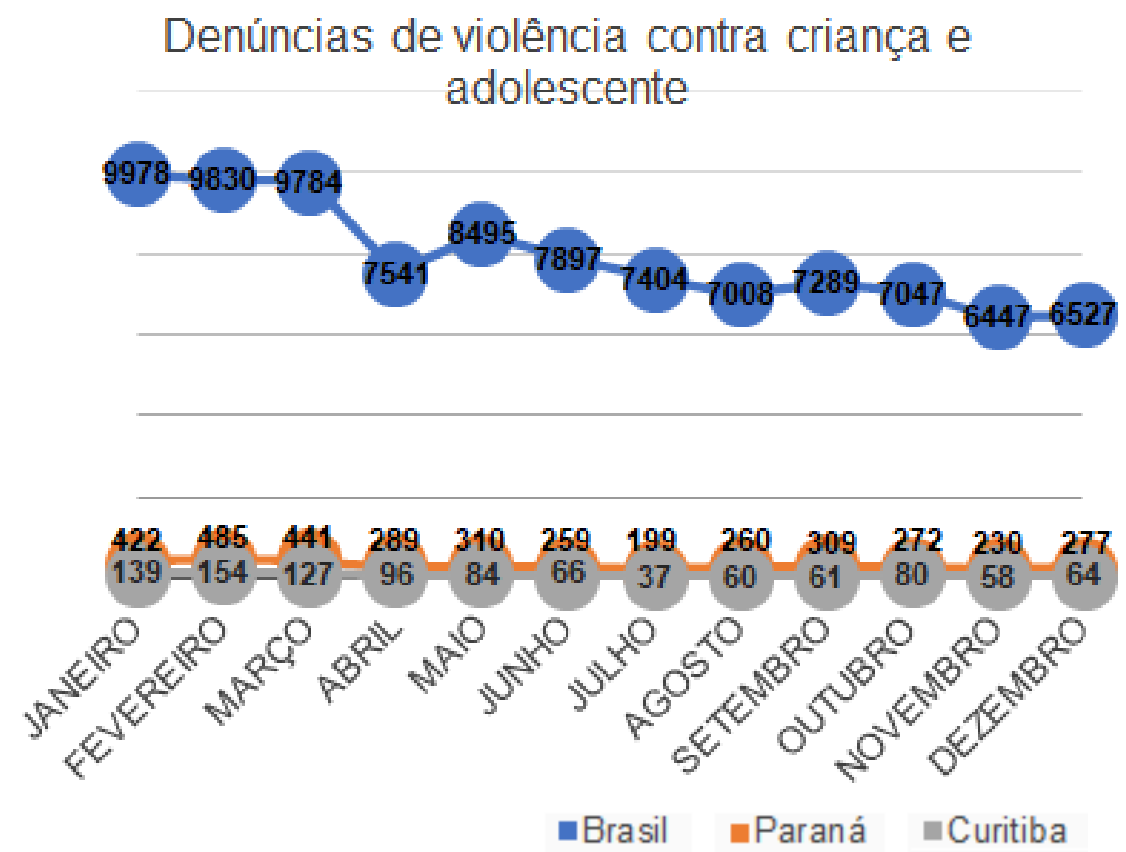

Fig. 1. Distribuição de denúncias por mês, 2020. (Fonte: Painel de Dados da Ouvidoria Nacional dos Direitos Humanos). 
Várias condições explicam a diminuição significativa das notificações tais como o medo de contaminação, o distanciamento imposto, a insegurança e ameaças intrafamiliares dificultam a denúncia e reduzem a procura de apoio (Platt, Guedert, \& Coelho, 2020).

Ademais, a queda das notificações e denúncias provavelmente reflete uma menor identificação e notificação dos casos existentes, em vez de representar redução do número de casos de violência contra crianças e adolescentes (Levandowski, et al, 2021).

Este paradoxo entre as medidas de distanciamento social e a maior exposição das crianças e adolescentes à violência doméstica, em especial, evidencia não somente mais uma contradição que a pandemia impõe, mas também a quão insuficiente e despreparada está a rede de proteção brasileira para o combate às violências. Além disso, traz à tona o descaso dos governos federal, estaduais e municipais em relação aos direitos sociais no Brasil e ao enfrentamento da crise sanitária da COVID-19.

\subsection{Caracterização da Violência contra as Crianças nos Programas e Políticas Públicas durante a Pandemia de COVID-19}

No que se refere aos tipos de violência perpetrados contra as crianças, na Figura 2 é possível verificar a distribuição no município de Curitiba.

\section{Tipos de violações}

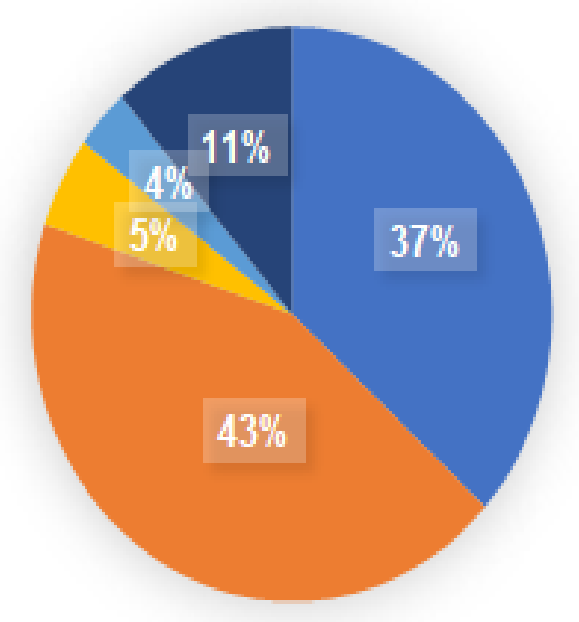

-Violência física

-Violência psicológica

च Agressőes que violam a honra

- Agressões que violam

0 direito e a liberdade

- Violações gerais

Fig. 2. Distribuição por tipo de violação contra a criança e o adolescente, Curitiba. Janeiro a junho, 2020. (Fonte: Painel de Dados da Ouvidoria Nacional dos Direitos Humanos).

Revisão sistemática de estudos longitudinais prospectivos identificou que a perda de emprego dos pais, por exemplo, é o principal motivo de violência psicológica e abuso físico (Conrad-Hiebner e Byram, 2020). Ressalta-se que a sobrecarga parental gera irritabilidade e tensão, refletindo no comportamento dos filhos, que passam a adotar atitudes semelhantes (Platt, Guedert, Coelho, 2020). Por conseguinte, as crianças são mais vulneráveis de acordo com o estresse psicossocial a que estão submetidas e estão em maior risco de abuso, sobretudo porque elas podem não entender a situação que está acontecendo na família diante das recessões socioeconômicas relacionadas ao COVID19 (Lawson, Piel, \& Simon, 2020).

Os dados declarados nas denúncias referentes ao sexo e à idade ajudam na caracterização do perfil das crianças e adolescentes em situação de violência na pandemia de COVID-19. O maior número de vítimas do sexo feminino está associado à desigualdade de gênero, que estabelece relações de poder distintas entre homens e mulheres. Dessa forma, é possível constatar a subalternidade de gênero desde a infância (Fornari, Sakata, Egry, \& da Fonseca, 2018). A Figura 3 exibe o quantitativo de vítimas por sexo para recém-nascido, crianças e adolescentes. 


\section{Violações por faixa etária e sexo}

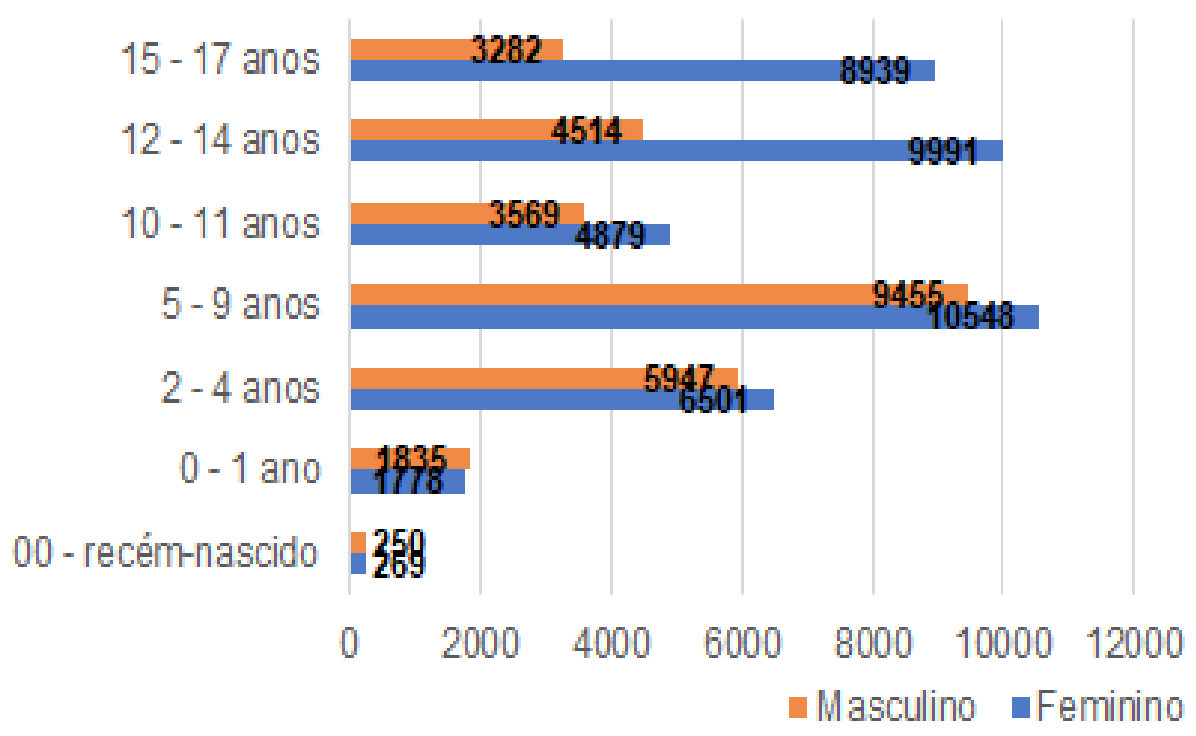

Fig. 3. Distribuição de violações por faixa etária da vítima e por sexo, Brasil. 2020. (Fonte: Painel de Dados da Ouvidoria Nacional dos Direitos Humanos).

O patriarcado e normas sociais sexistas implicam em maiores responsabilidades de cuidados não remunerados e, consequentemente, maior exposição aos trabalhos e aos estresses domiciliares, determinada pelo gênero. Assim, mulheres tendem à precarização da saúde mental, exemplificada na vulnerabilidade ao risco de violência. As meninas, antes da pandemia de COVID-19, gastavam $40 \%$ a mais do seu tempo do que os meninos em trabalho doméstico. Durante o isolamento social, dois terços delas fazem mais tarefas domésticas, e mais da metade relata gastar mais tempo cuidando dos irmãos. Além disso, o acesso a serviços de internet móvel é $26 \%$ menor para o sexo feminino em comparação com o masculino em todo o mundo, dificultando além das denúncias, o acesso à informação e à educação (Oxfam, 2021).

Evidencia-se que famílias de baixa renda residem em moradias com poucos cômodos ou desprovidas de condições adequadas de habitabilidade, assim o convívio entre os membros durante a pandemia é reforçado. Consequentemente, as restrições de movimento acentuam a sensação de poder e do controle pelos abusadores. Devido à relação de dependência da criança para sobreviver e ao convívio próximo com o adulto agressor, a realização de denúncia pela vítima é dificultada (Marques, et al, 2020).

Logo, medidas para melhorar a vigilância, identificar fatores de risco, desenvolver intervenções e aumentar a eficácia das políticas e dos programas devem ser implementadas para apoiar as pessoas mais vulneráveis em relação à violência doméstica, principalmente junto a adolescentes negros e meninas em comunidades pobres (Martins-Filho, Damascena, Lage, \& Sposato, 2020).

A vulnerabilidade afeta ações e intervenções de programas e políticas públicas (Levandowski, et al, 2021). Todavia, os preceitos institucionais sozinhos não asseguram apoio suficiente para as ações de notificação e prevenção, dessa forma, o trabalho em rede é necessário, nos níveis institucional e local onde se inserem os serviços.

No âmbito municipal, verificam-se empecilhos políticos e burocráticos para mobilizar recursos de poder e combater relações desiguais que resultam em violência contra crianças. Por isso, para o enfrentamento da violência doméstica durante e após a pandemia, são importantes políticas de articulação intersetorial - educação, saúde, assistência social e justiça - e que atuem na cultura de subordinação, viabilizando uma práxis transformadora (Egry, Apostolico, \& Morais, 2018). 
O presente estudo constatou que, no primeiro ano da pandemia da COVID-19, houve uma redução no número de denúncias associadas à violência contra crianças, acompanhada pelo número limitado de políticas e programas para o enfrentamento do problema.

Pesquisa revela que na pandemia de COVID-19 as crianças foram afetadas pela suspensão ou pelo acesso remoto a serviços de bem-estar, apoio social e familiar. Os autores também entendem que após o momento pandêmico a situação das crianças vulneráveis será agravada devido ao aumento da desigualdade de renda e da exclusão digital, interferindo no acesso a nutrição básica, abrigo, informação e educação (Raman, Harries, et. al 2020). Portanto, revela-se a necessidade de proposição e implementação de políticas e programas para dar respostas ao momento atual, bem como ao que se segue à crise sanitária.

Verifica-se também no presente estudo que as políticas e programas, mesmo que em primeira instância possam ser deflagrados por impacto quantitativo (como no caso das notificações e subnotificações em época de pandemia e reclusão social), requerem estudos de metodologia qualitativa. Estes conseguem decifrar melhor a realidade objetiva desde a intencionalidade das políticas até a transformação em programas e ações que visem à superação, neste estudo, da violência doméstica em tempos de pandemia.

Para este aprofundamento analítico é imperativo o uso de categorias sociais, tal como já mencionadas, e referentes à subalternidade de gênero, de geração e de raça-etnia, como parte integrante da interpretação crítico-dialético do processo saúde-doença, conhecido como determinação social do processo saúde-doença (Egry, 2013; Fornari 2018; SakataSo, 2016).

Os limites desta pesquisa documental foram relativos à dificuldade de acesso às fontes, e a não representatividade integral do fenômeno pesquisado. Como vantagens, a pesquisa documental garante a estabilidade dos dados no decorrer do tempo e como base para outros estudos, além do baixo custo na implementação da metodologia (Alves, Saramago, Valente, \& Sousa, 2021).

\section{Conclusão}

A instalação da pandemia da COVID-19 revelou a necessidade de respostas rápidas dos governos para os problemas subsequentes, bem como dos pesquisadores. Mas essas respostas não se encontram dadas, ao contrário, a situação da violência foi agravada pelas sub notificações. Por outro lado, revelou também o despreparo dos governos brasileiros na garantia dos direitos sociais e no enfrentamento das violências e da pandemia no país.

Políticas e programas precisam responder ao número de casos de violência doméstica contra a criança no período da pandemia de COVID-19, mas também reforçam a compreensão das vulnerabilidades sociais na perspectiva de gênero, classe social, raça e geração para a superação do problema tanto da violência quanto da própria pandemia.

A pesquisa documental de abordagem qualitativa mostrou-se potente para o entendimento do fenômeno social da violência doméstica contra a criança no contexto pandêmico, permitindo identificar, em uma primeira análise, alguns impactos que a pandemia causou no enfrentamento da violência doméstica contra as crianças, além da falta de maior assertividade nas políticas públicas para o enfrentamento do fenômeno. 


\section{Referências}

Alves, L. H., Saramago, G., Valente L. de F., \& Sousa, A. S. de. (2021). Análise documental e sua contribuição no desenvolvimento da pesquisa científica. Cadernos da Fucamp, 20(43), 51-63.

Bardin, L. (2011). Análise de Conteúdo. São Paulo: Edições 70.

Bradbury-Jones, C., \& Isham, L. (2020, July 1). The pandemic paradox: The consequences of COVID-19 on domestic violence. Journal of Clinical Nursing, 29(13-14), 2047-2049. https://doi.org/10.1111/jocn.15296

Cluver, L., Lachman, J. M., Sherr, L., Wessels, I., Krug, E., Rakotomalala, S., Blight, S., Hillis, S., Bachman, G., Green, O., Butchart, A., Tomlinson, M., Ward, C. L., Doubt, J., \& McDonald, K. (2020). Parenting in a time of COVID-19. The Lancet, 395(10231), e64. https://doi.org/10.1016/S0140-6736(20)30736-4

CLP Liderança Pública. (2019). Entendendo os conceitos básicos de Políticas Públicas. https://www.clp.org.br/entendendo-os-conceitos-basicos-mlg2-de-politicas-publicas-mlg2/

Conrad-Hiebner, A., \& Byram, E. (2020). The Temporal Impact of Economic Insecurity on Child Maltreatment: A Systematic Review. https://doi.org/10.1177/1524838018756122

Creswell J. W. (2014). Investigação qualitativa e projeto de pesquisa. (3th ed.). Editora Penso: Porto Alegre.

Curitiba. (2016). Conselho Municipal dos Direitos da Criança e do Adolescente e Conselho Municipal de Assistência. Plano Decenal Direitos Humanos de Crianças e Adolescentes de Curitiba. https://fas.curitiba.pr.gov.br/conteudo.aspx?idf=1255

Egry, E.Y., da Fonseca, R. M. G. S., \& Oliveira, M. A. de C. (2013). Ciência, Saúde Coletiva e Enfermagem: destacando as categorias gênero e geração na episteme da práxis. Revista Brasileira de Enfermagem, 66, 119-133.

Egry, E. Y. (1996). Saúde coletiva: construindo um novo método em enfermagem. São Paulo: Ícone Editora.

Egry, E. Y., Apostólico, M. R., \& Morais, T. C. P. (2018). Notificação da violência infantil, fluxos de atenção e processo de trabalho dos profissionais da atenção primária em saúde. Ciência e Saúde Coletiva, 23(1), 83-92. https://doi.org/10.1590/1413-81232018231.22062017

Fornari, L. F., Sakata-So, K. N., Egry, E. Y., \& da Fonseca, R. M. G. S. (2018). As perspectivas de gênero e geração nas narrativas de mulheres abusadas sexualmente na infância. Revista LatinoAmericana de Enfermagem, 26. https://doi.org/10.1590/1518-8345.2771.3078

Fundação Oswaldo Cruz. (2020). Crianças na pandemia COVID-19. https://www.fiocruzbrasilia.fiocruz.br/wp-content/uploads/2020/05/crianças_pandemia.pdf

Fundação Oswaldo Cruz. (2020). Violência doméstica e familiar na COVID-19. https://portal.fiocruz.br/sites/portal.fiocruz.br/files/documentos/saude-mental-e-atencaopsicossocial-na-pandemia-covid-19-violencia-domestica-e-familiar-na-covid-19.pdf

Gil, A. C. (2017). Como elaborar projetos de pesquisa (6th ed.). São Paulo: Atlas.

Instituto Brasileiro de Geografia e Estatística. (2020). Cidades. https://cidades.ibge.gov.br/

Instituto Paranaense de Desenvolvimento Econômico e Social. (2020). IPARDES. http://www.ipardes.gov.br/

Kripka, R. M. L., Scheller, M., \& Bonotto, D. de L. (2015). Pesquisa Documental: considerações sobre conceitos e características na Pesquisa Qualitativa. https://proceedings.ciaiq.org/index.php/ciaiq2015/article/view/252/248

Lawson, M., Piel, M. H., \& Simon, M. (2020). Child Maltreatment during the COVID-19 Pandemic: Consequences of Parental Job Loss on Psychological and Physical Abuse Towards Children. Child Abuse and Neglect, 110. https://doi.org/10.1016/j.chiabu.2020.104709

Levandowski, M. L., Stahnke, D. N., Munhoz, T. N., Von Hohendorff, J., \& Salvador-Silva, R. (2021). Impacto do distanciamento social nas notificações de violência contra crianças e adolescentes no Rio Grande do Sul, Brasil. Cadernos de Saúde Pública, 37(1). https://doi.org/10.1590/0102$311 \times 00140020$

Marques, E. S., de Moraes, C. L., Hasselmann, M. H., Deslandes, S. F., \& Reichenheim, M. E. (2020). A violência contra mulheres, crianças e adolescentes em tempos de pandemia pela COVID-19: panorama, motivações e formas de enfrentamento. Cadernos de Saúde Pública, 36(4). https://doi.org/10.1590/0102-311X00074420 
Martins-Filho, P. R., Damascena, N. P., Lage, R. C. M., \& Sposato, K. B. (2020). Decrease in child abuse notifications during COVID-19 outbreak: A reason for worry or celebration? Journal of Paediatrics and Child Health, 56(12), 1980-1981. https://doi.org/10.1111/jpc.15213

Minayo, M. C. de S. (2006). Violência e saúde (FIOCRUZ ed.). SciELO Books - Editora FIOCRUZ.

Minayo, M. C. de S., Deslandes, S. F., \& Gomes, R. (2007). Pesquisa social: teoria, método e criatividade. Vozes.

Minayo, M. C. S, Costa A.P. (2019). Técnicas que fazem uso da Palavra, do Olhar e da Empatia: Pesquisa qualitativa em ação. Aveiro: Ludomedia.

Ministério da Família da Mulher e dos Direitos Humanos. (2020). Painel de Dados da Ouvidoria Nacional de Direitos Humanos. https://www.gov.br/mdh/pt-br/ondh/paineldedadosdaondh

Ministério da Saúde. (2010). Linha de cuidado para a atenção integral à saúde de crianças, adolescentes e suas famílias em situação de violência. https://bvsms.saude.gov.br/bvs/publicacoes/linha_cuida

Ministério dos Direitos Humanos. (2018). Violência contra Crianças e Adolescentes: Análise de Cenários e Propostas de Políticas Públicas. https://www.gov.br/mdh/pt-br/centrais-de-conteudo/crianca-ead

Organização Mundial da Saúde. (2020). OMS afirma que COVID-19 é agora caracterizada como pandemia. https://www.paho.org/bra/index.php?option=com_content\&view=article\&id=6120:omsafirma-que-cov

Oxfam. (2021). O vírus da desigualdade. https://doi.org/10.21201

Platt, V. B., Guedert, J. M., \& Coelho, E. B. S. (2020). Violência contra crianças e adolescentes: notificações e alerta em tempos de pandemia. Revista Paulista de Pediatria, 39. https://doi.org/10.1590/1984-0462/2021/39/2020267

Raman, S., Harries, M., Nathawad, R., Kyeremateng, R., Seth, R., Lonne, B., Zwi, K., Spencer, N. J., Goldhagen, J., Mercer, R., Rubio, B., Tamburlini, G., Goldfeld, S., Woolfenden, S., Gander, S., \& Oberg, C. (2020). Where do we go from here? A child rights-based response to COVID-19. BMJ Paediatrics Open, 4(1). https://doi.org/10.1136/bmjpo-2020-000714

Secretaria Municipal da Saúde. (2021). Painel COVID-19 Curitiba. https://coronavirus.curitiba.pr.gov.br/painelcovid/

Secretaria Municipal da Saúde. (2017). Plano Municipal de Saúde de Curitiba 2018-2021. https://saude.curitiba.pr.gov.br/images/Plano Municipal de Saúde_SMS Curitiba_final 04.04.pdf

Silva, E. R. A. da, \& Oliveira, V. R. de. (2020). Proteção de crianças e adolescentes no contexto da pandemia da Covid-19: consequências e medidas necessárias para o enfrentamento. http://repositorio.ipea.gov.br/bitstream/11058/10041/1/NT_70_Disoc_Protecao de Criancas e Adolescentes no Contexto da Pandemia da Covid_19.pdf

Silveira, D. T., \& Códova, F. P. (2009). A pesquisa científica. In T. E. Gerhardt \& D. T. Silveira (Eds.), Métodos de Pesquisa (1st ed.). Universidade Federal do Rio Grande do Sul.

Sakata-So, K. N., Egry E. Y, Apostólico, M. R., \& Wazima, C. M. (2016). Vídeos institucionais podem contribuir ao debate para o enfrentamento da violência doméstica infantil? Ciência e Saúde Coletiva, 21(8), 2347-2356. https://doi.org.10.1590/1413-81232015218.04592016

The Alliance for Child Protection in Humanitarian Action. (2020). Technical Note: Protection of Children during the Coronavirus Pandemic (v.2). United Nations International Children's Fund.

United Nations Children's Fund. (2020). Protecting Children from Violence in the Time of COVID-19: Disruptions in prevention and response services. https://www.unicef.org/media/74146/file/Protecting-children-from-violence-in-the-time-of-covid19.pdf

Wollmann, A., Sato, E. S., Moreira, M. D., Cortiano, S., \& Rosa, T. E. A. (2020). Perfil das notificações de violência interpessoal/autoprovocada no município de Curitiba em 2019. https://saude.curitiba.pr.gov.br/images/RELATORIO2019notificaçõesdeviolencia.pdf

World Health Organization. (2020). Global status report on preventing violence against children 2020. https://violenceagainstchildren.un.org/sites/violenceagainstchildren.un.org/files/2020/global_status _report/global_status_report-eng.pdf

WHO Global. (2020). Addressing violence against children, women and older people during the COVID19 Pandemic: Key actions. World Health Organization. https://www.who.int/publications/i/item/WHO-2019-nCoV-Violence_actions-2020.1. 